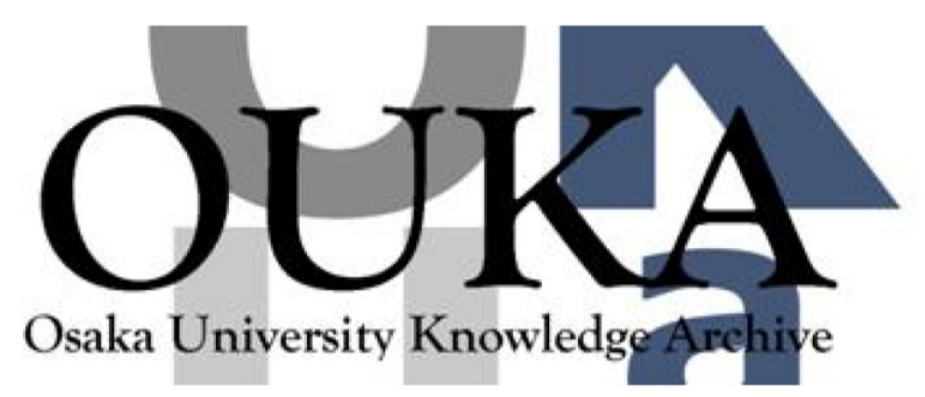

\begin{tabular}{|c|l|}
\hline Title & $\begin{array}{l}\text { Broad-range neutron spectra ident ification in } \\
\text { ultraintense laser interactions with carbon- } \\
\text { deuterated plasma }\end{array}$ \\
\hline Author(s) & Youssef, A.; Kodama, R.; Habara, H. et al. \\
\hline Citation & $\begin{array}{l}\text { Physics of Plasmas. 12(11) p. 110703-1-p. 110703- } \\
4\end{array}$ \\
\hline Issue Date & $2005-11$ \\
\hline oaire:version & VoR \\
\hline URL & https://hdl. handle. net/11094/2868 \\
\hline rights & \\
\hline Note & \\
\hline
\end{tabular}

Osaka University Knowledge Archive : OUKA

https://ir. Library. osaka-u. ac. jp/

Osaka University 


\title{
Broad-range neutron spectra identification in ultraintense laser interactions with carbon-deuterated plasma
}

\author{
A. Youssef ${ }^{\text {a) }}$ \\ Graduate School of Engineering, Osaka University, 2-1 Suita, Osaka 565-0871, Japan \\ R. Kodama, H. Habara, and K. A. Tanaka \\ Graduate School of Engineering, Osaka University, 2-1 Suita, Osaka 565-0871, Japan \\ and Institute of Laser Engineering, Osaka University, 2-6 Suita, Osaka 565-0871, Japan \\ Y. Sentoku \\ Department of Physics, University of Nevada, MS-220, Reno, Nevada 89557
}

M. Tampo and Y. Toyama

Institute of Laser Engineering, Osaka University, 2-6 Suita, Osaka 565-0871, Japan

(Received 23 June 2005; accepted 3 October 2005; published online 17 November 2005)

\begin{abstract}
Detailed neutron energy spectra produced from a CD2 target irradiated by a $450 \mathrm{fs}, 20 \mathrm{~J}, 1053 \mathrm{~nm}$ laser at an intensity of $3 \times 10^{18} \mathrm{~W} / \mathrm{cm}^{2}$ have been studied. Wide-ranging neutron spectra were observed from two different observation angles $20^{\circ}$ and $70^{\circ}$ relative to the rear-side target normal. The experiment and numerically calculated spectra, by a three-dimensional Monte Carlo code, indicate that the range of the measured spectra is larger than that produced by the $\mathrm{D}(d, n)^{3} \mathrm{He}$ reaction. An interpretation for the measured spectra is introduced by considering the ${ }^{12} \mathrm{C}(d, n){ }^{13} \mathrm{~N}$ and $\mathrm{D}\left({ }^{12} c, n\right){ }^{13} \mathrm{~N}$ reactions. In addition, the study revealed that the neutron spectra produced by the D-C and C-D reactions can overlap that produced by the D-D reaction, and due to their high cross sections, comparing to the D-D reaction, both of them effectively participate in the neutron yield. (C) 2005 American Institute of Physics. [DOI: 10.1063/1.2131847]
\end{abstract}

Recent progress in short-pulse laser technology has allowed focusing laser pulses with extremely high irradiation intensities. ${ }^{1}$ The interaction of this ultraintense femtosecond laser with solid targets acts as a source of energetic particles such as $\mathrm{MeV}$ electrons and ions. ${ }^{2}$ The fast ignitor concept, ${ }^{3}$ relevant to the inertial confinement fusion (ICF), enhances the interest in this process. Once the laser intensity exceeds $10^{18} \mathrm{~W} / \mathrm{cm}^{2}$, the generated hot electrons become relativistic. The propagation of this intense electron beam inside the target leads to generate $\gamma$ rays and accelerate ions which in turn initiate nuclear reactions producing neutrons, positrons, and radioactive nuclei. Whereas a relatively high number of papers are devoted to study hot electrons, $\gamma$ rays, and emitted ions, studies of femtosecond-laser-generated neutrons are rare and further detailed investigations are needed. Experimentally, neutron production has been observed by irradiating deuterated targets with ultraintense lasers at different intensities. ${ }^{4-11}$ The interpretation of the experimental results based on considering the $\mathrm{D}(d, n)^{3} \mathrm{He}$ reaction is the only productive reaction of the emitted neutron spectra. However, neutrons with energies lower than that produced by the D-D reaction (the absolute minimum energy of the D-D neutrons is $1.63 \mathrm{MeV}$ ) were observed when lasers with intensities of the order of $10^{18} \mathrm{~W} \mathrm{~cm}^{-2}$ is focused on solid CD2 targets. ${ }^{5,10}$ The identification of the reaction(s) that produce these neutrons is still needed. Such identification is crucial for ICF since the measured neutron yield is usually attributed to the

\footnotetext{
${ }^{a)}$ Author to whom correspondence should be addressed. Permanent address: Physics Department, Faculty of Science, South Valley University, Sohag 82524, Egypt. Electronic mail: ayoussef@ile.osaka-u.ac.jp
}

D-D reaction only. In this letter, we introduce for the first time a study that utilizes neutron spectroscopy to identify the generative reactions of a wide range of neutron spectra observed in ultraintense laser interactions with carbondeuterated plasma.

Figure 1 shows the schematic of the experiment which is performed using 30 TW GekkoMII (Ref. 12) short-pulse laser system at the Institute of Laser Engineering (ILE), Osaka University. The $450 \mathrm{fs}, 20 \mathrm{~J}, 1053 \mathrm{~nm}$ laser was focused on a CD2 target at an intensity of $3 \times 10^{18} \mathrm{~W} / \mathrm{cm}^{2}$. The incident angle of the $p$ polarized laser light was $20^{\circ}$ relative to the target normal. Two neutron detectors were positioned at 2.17 and $1.98 \mathrm{~m}$ from the target at angles $20^{\circ}$ and $70^{\circ}$ relative to the target normal, respectively. Neutron diagnostics consisting of current mode time-of-flight (TOF) detectors comprised a scintillator/photomultiplier tube (PMT) combination. The scintillator type, ultrafast timing plastic scintillator, is the quenched version of Bicron BC-422 scintillator (BC422Q). The scintillation light from BC-422Q has a rise time of less than 20 ps. ${ }^{13}$ A photomultiplier tube that is one of the highest performance timing tubes available is Hamamatsu R2083. It is an eight-stage PMT with a rise time of $700 \mathrm{ps}$ and a transit-time spread of $370 \mathrm{ps.} \mathrm{To} \mathrm{reduce} \mathrm{the} \mathrm{influence}$ of the undesirable flashes, cone-shaped collimators are installed in the chamber. The collimators limit the view of the detectors and reduce the bremsstrahlung flashes from the diagonal inner walls of the chamber. In addition, the detectors were shielded with $10-\mathrm{cm}$-thick $\mathrm{Pb}$ wall placed in the front and $5 \mathrm{~cm}$ of $\mathrm{Pb}$ plates on all sides. The modulation of the neutron spectra due to the shielding ${ }^{14}$ was taken into account. 


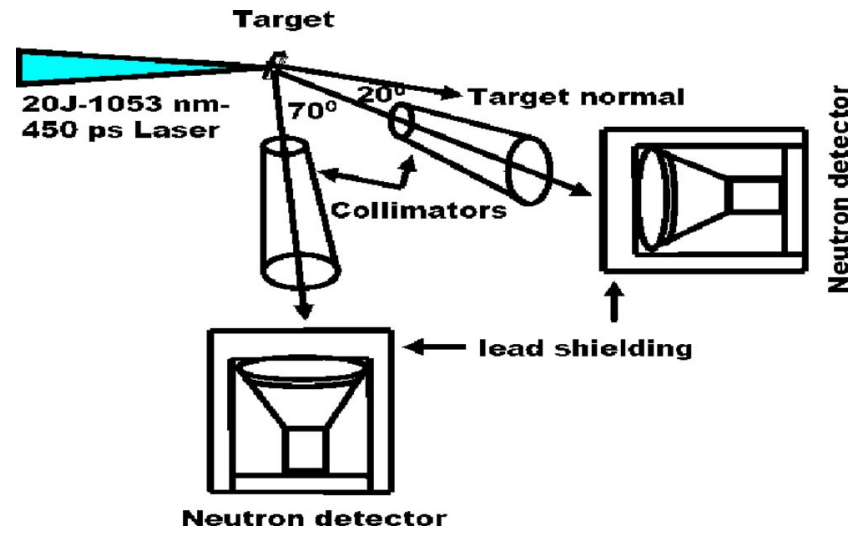

FIG. 1. View of the experimental setup. The $450 \mathrm{fs}, 20 \mathrm{~J}, 1053 \mathrm{~nm}$ laser was focused on a CD2 target at an intensity of $3 \times 10^{18} \mathrm{~W} / \mathrm{cm}^{2}$. Two neutron detectors were positioned at 2.17 and $1.98 \mathrm{~m}$ from the target at angles $20^{\circ}$ and $70^{\circ}$ relative to the target normal, respectively.

Therefore, time-of-flight values of the neutron groups were corrected by considering the delay of the neutrons inside the lead blocks. The accuracy of each energy value is calculated by using the differentiation method. ${ }^{15}$ In this method, the error in neutron energy is calculated by the partial differentiation depending on the experimental errors in both time of flight and distance of the detector from the target. The signal levels are normalized to the neutron yield per solid angle, taking into account the detector position and sensitivity.

Figure 2 shows the emitted neutron spectra observed in the forward direction from two angles of observation $20^{\circ}$ and $70^{\circ}$ relative to the target normal when a $5 \mu \mathrm{m} \mathrm{CD} 2$ target is irradiated by laser at an intensity of $3 \times 10^{18} \mathrm{~W} / \mathrm{cm}^{2}$. The neutron spectra observed at $20^{\circ}$ extend from $75 \pm 5 \mathrm{keV}$ up to $4.5 \pm 0.31 \mathrm{MeV}$ and those observed at $70^{\circ}$ extend from $20 \pm 1.4 \mathrm{keV}$ up to $4.0 \pm 0.28 \mathrm{MeV}$. This means that the energy range of the measured neutrons extends from $20 \pm 1.4 \mathrm{keV}$ up to $4.5 \pm 0.31 \mathrm{MeV}$.

The laboratory energy of the emitted neutron $E_{b}$ as a function of the incident ion energy $E_{a}$ and the angle $\theta$ between the incident ion and the emitted neutron ${ }^{16}$ are given as

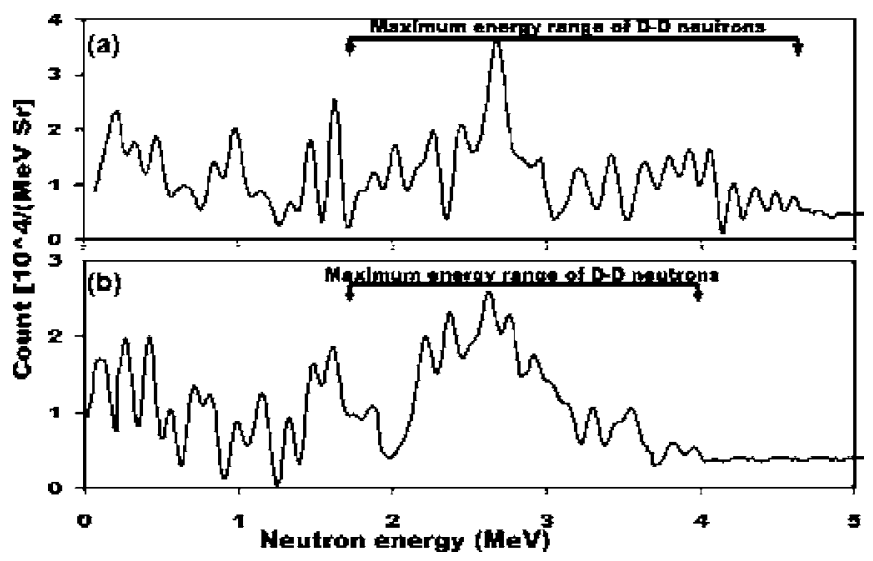

FIG. 2. The measured neutron spectra observed at (a) $20^{\circ}$ and (b) $70^{\circ}$. The spectra observed at $20^{\circ}$ extend from $75 \pm 5 \mathrm{keV}$ to $4.5 \pm 0.31 \mathrm{MeV}$ and that observed at $70^{\circ}$ extend from $20 \pm 1.4 \mathrm{keV}$ to $4.0 \pm 0.28 \mathrm{MeV}$. The two arrows display the maximum energy range of the D-D neutron spectra for each observation angle.

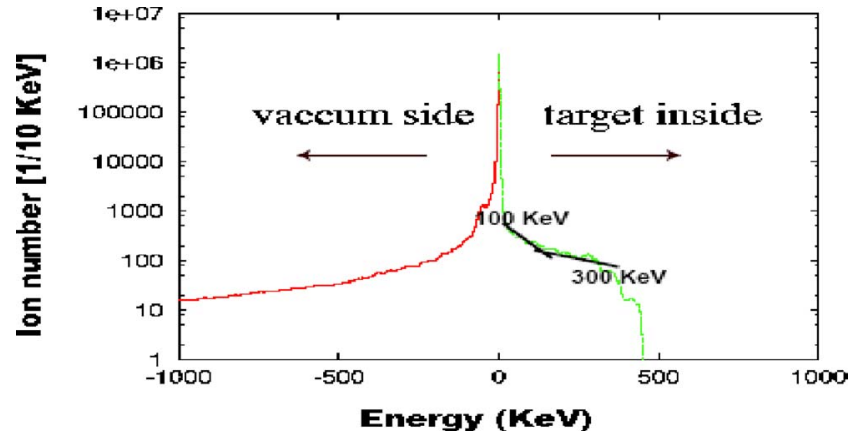

FIG. 3. (Color online). The energy spectrum of the accelerated deuterium ions inside the target from 2D PIC simulation. The energy spectrum can be fitted to two temperatures (100 and $300 \mathrm{keV})$. The bulk ions whose temperatures are very low $(\sim \mathrm{keV})$ and are not accelerated by the laser light are excluded from the fitting.

$$
\begin{aligned}
E_{b}^{1 / 2}= & \left(\left(m_{a} m_{b} E_{a}\right)^{1 / 2} \cos \theta \pm\left\{m_{a} m_{b} E_{a} \cos ^{2} \theta+\left(m_{Y}+m_{b}\right)\right.\right. \\
& \left.\left.\times\left[m_{Y} Q+\left(m_{Y}-m_{a}\right) E_{a}\right]\right\}^{1 / 2}\right) /\left(m_{Y}+m_{b}\right) .
\end{aligned}
$$

where $m_{a}, m_{b}$, and $m_{Y}$ are the masses of incident ion, emitted neutron, and residual nucleus, respectively. By considering beam fusion through $\mathrm{D}-\mathrm{D}$ reaction, the maximum energy of the emitted neutrons (in the forward direction $\theta=0$ ) will be $4.5 \pm 0.31 \mathrm{MeV}$ if the maximum energy of the accelerated $\mathrm{D}$ ions is $1.3 \pm 0.22 \mathrm{MeV}$. In this case, when the incident $\mathrm{D}$ ions are accelerated up to $1.3 \pm 0.22 \mathrm{MeV}$, the minimum energy of the produced neutrons by the D-D reaction (in the backward direction $\theta=180)$ is $1.7 \pm 0.12 \mathrm{MeV}$. Hence the maximum range of the neutron spectra produced by the $\mathrm{D}-\mathrm{D}$ reaction extends from $1.7 \pm 0.12$ up to $4.5 \pm 0.31 \mathrm{MeV}$ (the arrows in Fig. 2 display the maximum range for each observation angle). However, the observed neutron spectra contain neutrons with lower energies up to $20 \pm 1.4 \mathrm{keV}$. Consequently, the observed spectra cannot be explained by considering the D-D reaction only.

To elucidate the observed spectra, the energy spectrum of the accelerated deuterium ions inside the target in ultraintense laser plasma interactions has been investigated by twodimensional particle in cell simulation PICLS2D (Fig. 3). In the simulation, a $p$-polarized $1-\mu \mathrm{m}$ laser light with a pulse length of $500 \mathrm{fs}$ irradiates the deuteron plasma at an incidence angle of $20^{\circ}$. The critical density $n_{c}$ of this laser is $10^{21} \mathrm{~cm}^{-3}$. The laser has a peak intensity of 4 $\times 10^{18} \mathrm{~W} / \mathrm{cm}^{2}$ in a $10 \mu \mathrm{m}$ spot of a Gaussian profile. The target is a fully ionized deuteron plasma (ion mass $M$ $=3680 m_{e}$ ) with a peak density of $40 n_{c}$. A steep preplasma whose peak density is $4 n_{c}$ is placed in front of the target. It is extending in $2 \mu \mathrm{m}$ from the target surface exponentially with $0.66 \mu \mathrm{m}$ scale length. The plasma density at the expansion edge is $0.17 n_{c}$. The PIC simulation shows that the energy spectrum of ions accelerated into the target can be fitted to two temperatures $(100$ and $300 \mathrm{keV})$. The cutoff energy of the accelerated ions is about $500 \mathrm{keV}$, which is well explained by the sweeping acceleration due to the pondermotive pressure. ${ }^{17}$ This maximum deuteron energy can explain the generation of neutrons with energies up to $3.5 \mathrm{MeV}$ [Eq. (1)]. However, the ion energy might increase to become two 


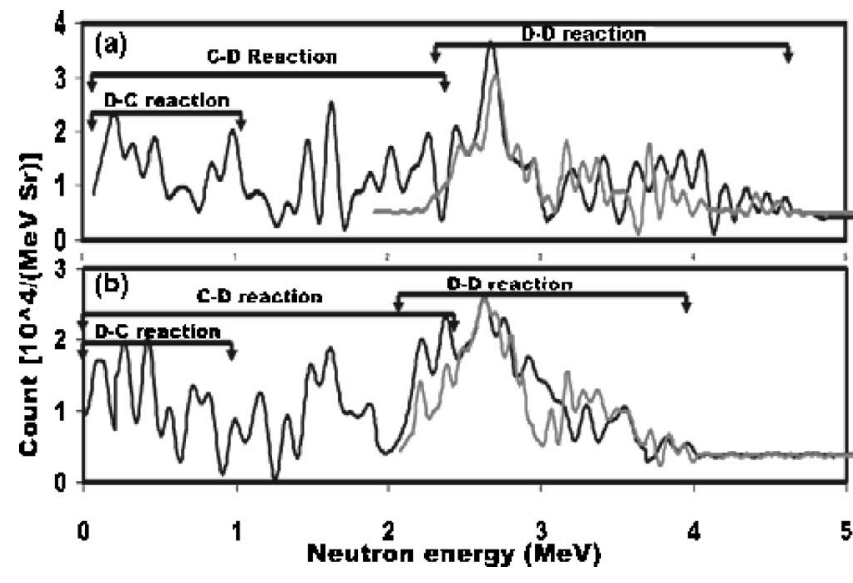

FIG. 4. Comparison between the measured neutron spectra (black) and the calculated spectra for the D-D reaction by 3D Monte Carlo code (gray) at (a) $20^{\circ}$ and (b) $70^{\circ}$. The D-D reaction can introduce an acceptable interpretation for the measured neutron spectra from $2.3 \pm 0.16$ up to $4.5 \pm 0.31 \mathrm{MeV}$ for the neutrons observed at $20^{\circ}$ and from $2.1 \pm 0.14$ up to $4 \pm 0.28 \mathrm{MeV}$ for those observed at $70^{\circ}$. The arrows display the produced neutron spectra by the $\mathrm{D}-\mathrm{D}$ reaction and the maximum energy ranges of the neutron spectra produced by the $\mathrm{D}-{ }^{12} \mathrm{C}$ and ${ }^{12} \mathrm{C}-\mathrm{D}$ reactions.

times or more in a gentle sloop preplasma as discussed in Ref. 17.

Furthermore, we performed numerical experiments by using three-dimensional (3D) Monte Carlo code. The code calculates the spectra of neutrons produced by $\mathrm{D}-\mathrm{D}$ reaction in a finite thickness target and emitted in specific directions of observation. For the best fitting to the experimental results, ions are assumed to be accelerated in the target normal direction ${ }^{9}$ with Maxwellian energy distribution. The distribution has two temperatures, 100 and $300 \mathrm{keV}$, that are in agreement with the PIC simulation. The multiple scattering of the accelerated ions due to small angle scattering by collisions with electrons was calculated by using Jackson's equation. ${ }^{18}$ The effect of this scattering on the path of the accelerated deuterons must be small but it is taken into account for the sake of high degree of accuracy. The neutron yield $Y$ emitted in a specific direction is calculated as

$$
Y=\int n_{1} n_{2} \sigma(v) v d t d v
$$

where $n_{1}$ is the number density of the accelerated ions, $n_{2}$ is the number of the target ions per unit volume, $\sigma(v)$ is the differential cross section of the nuclear reaction for a given value of energy, and $v$ is the velocity of the accelerated ions. The differential cross section $\sigma(E, \theta)$ for a given energy $E$ and emission angle $\theta$ calculated from experimental data and the best fitting calculated values ${ }^{19}$ by using Legendre polynomials are as follows:

$$
\sigma(E, \theta)=\sigma(E, 0) \Sigma A_{i} P_{i}(\theta),
$$

where $\sigma(E, 0)$ is the differential cross section for a given energy $E$ and zero emission angle, $P_{i}$ are Legendre coefficients, and $A_{i}$ are constants provided that $\Sigma A_{i}=1$. The calculated spectra fit well with the measured ones (Fig. 4) when the ion energy distribution in the Monte Carlo code reflects the exact distribution displayed in the PIC simulation (Fig.
3). In the same time, the degree of fitting becomes lower elsewhere when the previous condition is not fulfilled.

The 3D Monte Carlo simulation pointed out that the neutron spectra produced by the $\mathrm{D}-\mathrm{D}$ reaction are those produced in the range of $2.3 \pm 0.16-4.5 \pm 0.31 \mathrm{MeV}$ for the observed neutrons at $20^{\circ}$ and in the range of $2.1 \pm 0.14-4 \pm 0.28 \mathrm{MeV}$ for the observed neutrons at $70^{\circ}$ (Fig. 4). To identify the neutron spectra that emitted with lower energies, the other probable neutron productive reactions have to be investigated. These reactions include deuteron break up, deuteron electrodisintegration, and the interactions of the accelerated deuterons with ${ }^{13} \mathrm{C}$ ions (inside the target) and with the chamber wall (Al). In addition, photonuclear reactions and the interactions of protons (come from the contaminations on the target surface) with $\mathrm{D}$ and $\mathrm{C}$ ions inside the target and with the chamber wall ( $\mathrm{Al}$ ) have to be discussed. Finally the reactions between $\mathrm{D}$ ions and ${ }^{12} \mathrm{C}$ ions have to be considered.

Deuteron breakup cannot occur because its threshold energy is $4.45 \mathrm{MeV}$ whereas the maximum acceleration energy of the deuterons is $1.3 \pm 0.22 \mathrm{MeV}$. Deuteron electrodisintegration is negligible due to its extremely small cross section, $\sim 10 \mu \mathrm{b}$. ${ }^{20}$ The reactions between accelerated ions and ${ }^{13} \mathrm{C}$ ions can be ignored because of the small amount $(1 \%)$ of ${ }^{13} \mathrm{C}$ in the natural carbon even though the cross section is high. The interactions between the emitted deuterons and the chamber wall (Al) take place only when the deuterons have energies higher than $5.75 \mathrm{MeV}$.

Photonuclear reactions produced by the hard bremsstrahlung $\mathrm{x}$ rays or $\gamma$ rays are generated due to the stopping power of the relativistic electrons through the target. To effectively stop the relativistic electrons, CD2 target with thickness more than $300 \mu \mathrm{m}$ is needed. ${ }^{21}$ The thickness of our target is $5 \mu \mathrm{m}$ and hence the energies of the generated photons are too weak to produce any photonuclear reactions.

Protons (that contaminate the target surface) can produce neutrons from the interactions with deuterium and carbon ions inside the target if they accelerated to energies $\geqslant 3.34 \mathrm{MeV}$. As the charge-to-mass ratio of proton ion is twice that of deuterium ion, the estimated maximum energy of the accelerated proton ions would be twice that of the

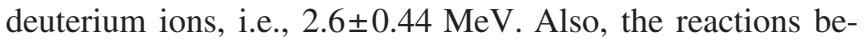
tween energetic protons and the chamber wall (Al) cannot produce neutrons unless protons accelerated to energies higher than $5.80 \mathrm{MeV}$.

The reactions between $\mathrm{D}$ ions and ${ }^{12} \mathrm{C}$ ions produce lowenergy neutrons, comparing to the D-D reaction, with high cross sections. The reaction between accelerated $\mathrm{D}$ ions and target background ${ }^{12} \mathrm{C}$ ions $\left(\mathrm{D}+{ }^{12} \mathrm{C} \rightarrow{ }^{13} \mathrm{~N}+n-0.28 \mathrm{MeV}\right)$ is possible as soon as the kinetic energy of the incident $\mathrm{D}$ ions exceeds the reaction threshold energy (i.e., $0.328 \mathrm{MeV}$ ). Comparing to the $\mathrm{D}-\mathrm{D}$ reaction, the $\mathrm{D}-{ }^{12} \mathrm{C}$ reaction has a very high cross section. While the maximum value of the D$\mathrm{D}$ reaction cross section is around $100 \mathrm{mb}$ within the energy range of $0-5 \mathrm{MeV},{ }^{19,22}$ that of the $\mathrm{D}-{ }^{12} \mathrm{C}$ reaction is around $250 \mathrm{mb}^{23}$ Because the deuterium ions are accelerated up to $1.3 \pm 0.22 \mathrm{MeV}$, the $\mathrm{D}-{ }^{12} \mathrm{C}$ reaction can produce neutrons with energies up to $1.02 \pm 0.23 \mathrm{MeV}$ [Eq. (1)] as shown in Fig. 4. Moreover, carbon ions can be accelerated to the same 
maximum velocity as deuterium ions. ${ }^{11,21}$ Thus, the maximum energy of ${ }^{12} \mathrm{C}$ ions can be six times (i.e., $7.8 \pm 1.3 \mathrm{MeV}$ ) that of $\mathrm{D}$ ions. Carbon ions that have energies $\geqslant 1.96 \mathrm{MeV}$ (the threshold energy of the ${ }^{12} \mathrm{C}-\mathrm{D}$ reaction) can produce neutrons through the interaction with the target background $\mathrm{D}$ ions. As the maximum energy of the incident ${ }^{12} \mathrm{C}$ ions is $7.8 \pm 1.3 \mathrm{MeV}$, the ${ }^{12} \mathrm{C}-\mathrm{D}$ reaction can produce neutrons with energies up to $2.4 \pm 0.39 \mathrm{MeV}$ [Eq. (1)] as shown in Fig. 4.

It is known that the cross sections of the $\mathrm{D}-{ }^{12} \mathrm{C}$ and ${ }^{12} \mathrm{C}-\mathrm{D}$ reactions are the same in the center-of-mass system. In the laboratory system, when ${ }^{12} \mathrm{C}$ ions have the same velocity as $\mathrm{D}$ ions, the cross section of the ${ }^{12} \mathrm{C}-\mathrm{D}$ reaction becomes approximately twice ${ }^{21}$ that of the $\mathrm{D}-{ }^{12} \mathrm{C}$ reaction. The endothermic reactions such as the $\mathrm{D}-{ }^{12} \mathrm{C}$ and ${ }^{12} \mathrm{C}-\mathrm{D}$ reactions produce neutrons with energies starting from extremely small value (theoretically zero) extending to a maximum value determined by the maximum energy of the incident ions. For example, in the case of deuterium ions accelerated inside the target up to $3 \mathrm{MeV}\left({ }^{12} \mathrm{C}\right.$ ions will be six times), the neutron spectra of the $\mathrm{D}-{ }^{12} \mathrm{C}$ reaction will extend to $2.7 \mathrm{MeV}$ and that of the ${ }^{12} \mathrm{C}-\mathrm{D}$ reaction will enlarge beyond $5 \mathrm{MeV}$ [Eq. (1)]. Then, these spectra will overlap the D-D reaction spectra. In the same time, due to their high cross sections, the $\mathrm{D}-{ }^{12} \mathrm{C}$ and ${ }^{12} \mathrm{C}-\mathrm{D}$ reactions will strongly take part in the total neutron yield. These results are reasonably consistent with the prediction of kinetic numerical simulations by Toupin et al. ${ }^{21}$

Unfortunately the differential cross-section data of the $\mathrm{D}-{ }^{12} \mathrm{C}$ and ${ }^{12} \mathrm{C}-\mathrm{D}$ reactions are not available. Accordingly, the produced spectra from these reactions could not be calculated for the same observation angles as the measured ones. However, the total neutron yields produced by the reactions D-D, D- ${ }^{12} \mathrm{C}$, and ${ }^{12} \mathrm{C}-\mathrm{D}$ have been estimated from Eq. (2) by using the total cross-section values and the total number of the accelerated $\mathrm{D}$ and ${ }^{12} \mathrm{C}$ ions. From the $3 \mathrm{D}$ Monte Carlo simulation, the best fitting to the experimental results are obtained when the number of the accelerated D ions was $2 \times 10^{12} / \mathrm{sr}$. This means that the total number of the accelerated $\mathrm{D}$ ions is close to $2 \times 10^{13}$ and the expected total number of the accelerated ${ }^{12} \mathrm{C}$ ions $(50 \%$ of the number of $\mathrm{D}$ ions) is around $10^{13}$. The estimated total neutron yield (in the $4 \Pi$ space) of the D-D reaction is $9 \times 10^{5}$, whereas those of the $\mathrm{D}-{ }^{12} \mathrm{C}$ and ${ }^{12} \mathrm{C}-\mathrm{D}$ reactions are $2.3 \times 10^{5}$ and 4.1 $\times 10^{5}$, respectively.

The total energy $\left(E_{t}\right)$ of the accelerated ions can be calculated as follows:

$$
E_{t}=\int F_{\mathrm{MB}} g(E) d E,
$$

where $F_{\mathrm{MB}}$ is the Maxwell-Boltzmann distribution function and $g(E)$ is the density of states. The calculated total energy of the accelerated $\mathrm{D}$ ions is $0.5 \mathrm{~J}$ which is equivalent to $2.5 \%$ of the laser energy. The energy of the full stripping carbon ion can be six times that of $\mathrm{D}$ ion and the number of ${ }^{12} \mathrm{C}$ ions is $50 \%$ of the number of $\mathrm{D}$ ions. Under these assumptions, the utmost laser-energy transfer to ${ }^{12} \mathrm{C}$ ions would be $7.5 \%$.
Then the maximum converted laser energy to ions might be $10 \%$ or less.

In summarize, we have studied the creative neutron reactions when an ultraintense laser at an intensity of 3 $\times 10^{18} \mathrm{~W} / \mathrm{cm}^{2}$ is focused on a CD2 target. The experimental results are compared to 3D Monte Carlo code and the energy spectrum of the accelerated D ions inside the target has been investigated by 2D PIC simulation. Neutrons with energies lower than that produced by the D-D reaction were observed in the measured neutron spectra. By considering the $\mathrm{D}-{ }^{12} \mathrm{C}$ and ${ }^{12} \mathrm{C}-\mathrm{D}$ reactions, a complete explanation for the measured neutron spectra is introduced. With increasing the energy of the accelerated ions, neutron spectra produced by the $\mathrm{D}-{ }^{12} \mathrm{C}$ and ${ }^{12} \mathrm{C}-\mathrm{D}$ reactions can effectively overlap that produced by the D-D reaction. Due to the high cross sections of the $\mathrm{D}-{ }^{12} \mathrm{C}$ and ${ }^{12} \mathrm{C}-\mathrm{D}$ reactions, their neutron yields strongly participate in the total neutron yield. We have to keep in mind that more reactions will contribute in the neutron production when using higher energy and intensity lasers. These results are of critical importance for laser fusion in which the total neutron yield is usually attributed to the D-D reaction only.

We are grateful to the members of the Laser, Target, and Measurement Tech. for their technical assistance.

${ }^{1}$ M. D. Perry, D. Pennington, B. C. Stuart et al., Opt. Lett. 24, 160 (1999).

${ }^{2}$ G. Malka and J. L. Miquel, Phys. Rev. Lett. 77, 75 (1996).

${ }^{3}$ M. Tabak, J. Hammer, M. E. Glinsky et al., Phys. Plasmas 1, 1626 (1994).

${ }^{4}$ P. A. Norreys, A. P. Fews, F. N. Beg et al., Plasma Phys. Controlled Fusion 40, 175 (1998).

${ }^{5}$ G. Pretzler, A. Saemann, A. Pukhov et al., Phys. Rev. E 58, 1165 (1998).

${ }^{6}$ R. Kodama, K. A. Tanaka, T. Yamanaka et al., Plasma Phys. Controlled Fusion 41, A419 (1999).

${ }^{7}$ L. Disdier, J-P. Graconnet, G. Malka et al., Phys. Rev. Lett. 82, 1454 (1999).

${ }^{8}$ K. A. Tanaka, R. Kodama, H. Fujita et al., Phys. Plasmas 7, 2014 (2000). ${ }^{9}$ H. Habara, R. Kodama, Y. Sentoku et al., Phys. Rev. E 69, 036407 (2004); Phys. Plasmas 10, 3712 (2003).

${ }^{10}$ D. Hilscher, O. Berndt, M. Enke et al., Phys. Rev. E 64, 016414 (2001).

${ }^{11}$ N. Izumi, Y. Sentoku, H. Habara et al., Phys. Rev. E 65, 036413 (2002).

${ }^{12}$ Y. Kitagawa, R. Kodama, K. Takahashi et al., Fusion Eng. Des. 44, 261 (1999).

${ }^{13}$ R. A. Lerche and D. W. Phillion, Conference Record of the 1991 IEEE Nuclear Science Symposium and Medical Imaging Conference, Santa Fe, New Mexico, 2-9 November 1991 (IEEE, Piscataway, NJ, 1991), Vol. I, 91CH3100-5.

${ }^{14}$ R. J. Leeper and J. Chang, IEEE Trans. Nucl. Sci. NS-29, 798 (1982).

${ }^{15}$ G. F. Knoll, Radiation Detection and Measurement, 2nd ed. (Wiley, New York, 1989).

${ }^{16}$ D. Halliday, Introductory Nuclear Physics, 2nd ed. (Wiley, New York, 1955).

${ }^{17}$ Y. Sentoku, T. E. Cowan, A. J. Kemp, and H. Ruhl, Phys. Plasmas 10, 2009 (2003)

${ }^{18}$ J. D. Jackson, Classical Electrodynamics, 3rd ed. (Wiley, New York, 1998).

${ }^{19}$ H. Liskin and A. Paulsen, Nucl. Data Tables 11, 569 (1973).

${ }^{20}$ D. Harder, R. Mehling, and A. C. England, Phys. Lett. 32B, 610 (1970).

${ }^{21}$ C. Toupin, E. Lefebvre, and G. Bonnaud, Phys. Plasmas 8, 1011 (2001).

${ }^{22}$ A. Krauss, H. W. Becker, H. P. Trautvetter, C. Rolfs, and K. Brand, Nucl. Phys. A 465, 150 (1987).

${ }^{23}$ R. W. Michelmann, J. Krauskopf, J. D. Meyer, and K. Bethge, Nucl. Instrum. Methods Phys. Res. B 51, 1 (1990); C. R. Brune and R W. Kavanagh, Phys. Rev. C 45, 1382 (1992). 